\title{
$\left.\operatorname{AIP}\right|_{\mid \text {Fysids of }} ^{\text {Phy }}$
}

\section{Vortex rings impinging on permeable boundaries}

Anna Mujal-Colilles, Stuart B. Dalziel, and Allen Bateman

Citation: Physics of Fluids (1994-present) 27, 015106 (2015); doi: 10.1063/1.4906504

View online: http://dx.doi.org/10.1063/1.4906504

View Table of Contents: http://scitation.aip.org/content/aip/journal/pof2/27/1?ver=pdfcov

Published by the AIP Publishing

\section{Articles you may be interested in}

Interaction of vortex rings with multiple permeable screens

Phys. Fluids 26, 113101 (2014); 10.1063/1.4900769

Laser energy deposition effectiveness on shock-wave boundary-layer interactions over cylinderflare combinations

Phys. Fluids 26, 096103 (2014); 10.1063/1.4896288

Resuspension onset and crater erosion by a vortex ring interacting with a particle layer Phys. Fluids 24, 063301 (2012); 10.1063/1.4716000

The effective slip length and vortex formation in laminar flow over a rough surface Phys. Fluids 21, 052105 (2009); 10.1063/1.3121305

Vortex ring pinchoff in the presence of simultaneously initiated uniform background co-flow Phys. Fluids 15, L49 (2003); 10.1063/1.1584436

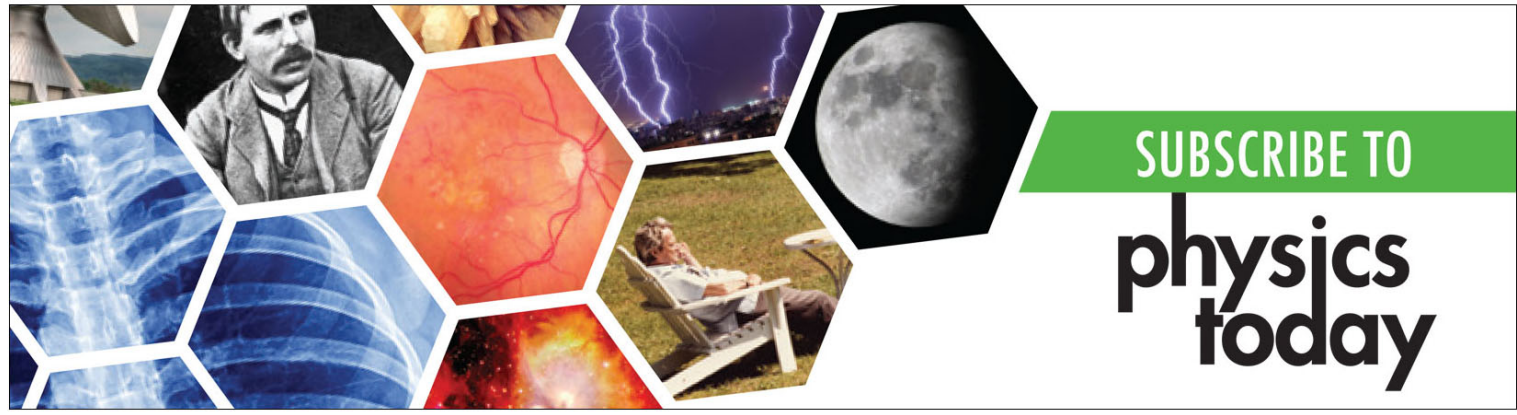




\title{
Vortex rings impinging on permeable boundaries
}

\author{
Anna Mujal-Colilles, ${ }^{1, a)}$ Stuart B. Dalziel, ${ }^{2}$ and Allen Bateman ${ }^{3}$ \\ ${ }^{1}$ Marine Engineering Laboratory, Barcelona Tech - UPC, Barcelona, Spain \\ ${ }^{2}$ Department of Applied Mathematics and Theoretical Physics, Cambridge University, \\ Cambridge, United Kingdom \\ ${ }^{3}$ Sediment Transport Research Group, Barcelona Tech - UPC, Barcelona, Spain
}

(Received 5 June 2014; accepted 11 January 2015; published online 27 January 2015)

\begin{abstract}
Experiments with vortex rings impinging permeable and solid boundaries are presented in order to investigate the influence of permeability. Utilizing Particle Image Velocimetry, we compared the behaviour of a vortex ring impinging four different reticulated foams (with permeability $k \sim 26-85 \times 10^{-8} \mathrm{~m}^{2}$ ) and a solid boundary. Results show how permeability affects the stretching phenomena of the vortex ring and the formation and evolution of the secondary vortex ring with opposite sign. Moreover, permeability also affects the macroscopic no-slip boundary condition found on the solid boundary, turning it into an apparent slip boundary condition for the most permeable boundary. The apparent slip-boundary condition and the flux exchange between the ambient fluid and the foam are jointly responsible for both the modified formation of the secondary vortex and changes on the vortex ring diameter increase. (C) 2015 AIP Publishing LLC. [http://dx.doi.org/10.1063/1.4906504]
\end{abstract}

\section{INTRODUCTION}

Vortex rings form spontaneously in many unsteady processes found in nature. Volcanic eruptions, swimming squid, starting jets, and some dolphin games all involve structures taking the form of vortex rings. Some industrial processes use the impingement of a vortex ring onto a solid surface to dislodge the particles that can be trapped in it (see Ref. 1), and vortex rings are a serious issue when landing a helicopter (e.g., Refs. 2 and 3).

The first analysis of a vortex ring structure was described by Lord Kelvin ${ }^{4}$ for vortex rings with a very thin core compared to the ring diameter. At the opposite limit, Hill ${ }^{5}$ detailed the characteristics of a vortex ring with the core diameter equal to the radius of a vortex ring, a structure now known as the Hill's spherical vortex. Batchelor ${ }^{6}$ described vortex rings as a single circular line vortex for inviscid fluids where the core was infinitesimally small and the propagation velocity was infinite. Subsequently, Norbury ${ }^{7}$ proposed a theoretical expression for vortex rings with a thin size of the core and a finite velocity of propagation and extended this to the entire range of vortex rings with different core sizes. Maxworthy ${ }^{8}$ carried out a series of experiments with different vortex ring formation characteristics to study its influence on the velocity of propagation, the core size, and the existence of instabilities; his studies revealed the existence of entrainment causing vortex deceleration.

A model for the canonical case of a vortex ring impinging a perpendicular solid wall was proposed by Saffman, ${ }^{9}$ using the mirroring of a vortex pair moving towards a symmetric vortex pair (with the plane of symmetry perpendicular to the direction of the motion). Cerra and Smith ${ }^{10}$ and Walker et al. ${ }^{11}$ pioneered the experimental study of the vortex ring impacting on a solid boundary, with Orlandi and Verizcco ${ }^{12}$ and Swearingen et al.$^{13}$ undertaking some of the earliest simulations. They all found a stretching of the core when approaching the wall, an increase in the diameter of the vortex ring, and the existence of a rebound of the core parallel to the formation of a secondary vortex with opposite sign. ${ }^{14}$

a)Anna.mujal@upc.edu 
More recently, attention has turned to the possibility of resuspension due to a vortex ring impacting a bed of particles (e.g., Refs. 1 and 15-18). Of particular interest here is the suggestion by Bethke and Dalziel ${ }^{19}$ that the permeability/porosity of the sediment bed may influence the dynamics of the interaction.

In previous studies, the interaction of vortex rings with porous boundaries has been related mainly to thin permeable grids with different porosities and wire diameter. Adhikari and Lim ${ }^{20}$ and Naaktgeboren $e t a l .{ }^{21}$ compared the impact of a thin porous grid on the vortex ring propagation with the interaction with a solid wall, varying mainly the Reynolds number and the grid porosity (defined as the ratio between the void spaces and the total area of the grid). They found that porosity influenced the increase of the vortex ring diameter: rings impinging higher porosity grids did not increase their diameter while approaching the grid. Moreover, the existence of the secondary vortex cores disappeared and the vortex ring was transmitted through the grids. Hryunk et al. ${ }^{22}$ showed how the scales of the grid also influenced the vortex/grid interaction. In particular, they studied constant porosity grids with variable wire diameter using constant Reynolds number vortex rings, and showed how the propagation of the ring beyond the grid was influenced by the length scales of the grid.

The work presented herein focuses on the interaction of vortex rings with thick permeable boundaries, relative to the core diameter, overlying impermeable base. This research aims to explore the influence of such boundaries on the vortex ring propagation.

This paper is organized as follows. The experimental methods and basic configuration are introduced in Sec. II, while Sec. III presents the main experimental results. These results are discussed in Sec. IV and finally, conclusions are presented in Sec. V.

\section{MATERIALS AND METHODS}

The experiments were carried out using a 361 acrylic tank, essentially the same as that described by Refs. 17 and 19. The tank has a square base $(300 \times 300 \mathrm{~mm})$, and a $400 \mathrm{~mm}$ height. The front face was left completely transparent, while the bottom and two lateral faces were covered on the inside with matt black plastic film to avoid the influence of ambient light; the third vertical face was covered with the same film except for a narrow vertical slot to allow illumination by a thin light sheet (see Figure 1). The lower boundary was either solid (using the base of the tank), or porous (using blocks of reticulated polyether foam cut to fit within the tank; see below). In either case, the tank was always filled to a depth of $300 \mathrm{~mm}$ above the top of the porous/permeable boundary: this is the bottom of the tank in the solid boundary experiments and the top of the porous layer in permeable boundary cases. The tank was filled with a column of salty water $\left(\rho=1020 \mathrm{~kg} / \mathrm{m}^{3}\right)$ to ensure the particles used for measuring the velocity field were neutrally buoyant.

The vortex ring was created in the same manner as used by Refs. 17 and 19. In particular, a polyvinyl chloride (PVC) tube of internal diameter $D_{t}=39 \mathrm{~mm}$ was submerged to a depth of $70 \mathrm{~mm}$ beneath the surface of the water. A slug of water was driven out the end of the tube by introducing air from a bicycle "track pump." This pump, with internal diameter $D_{s}=29 \mathrm{~mm}$, was actuated by an electric motor connected to its handle via a piece of nylon cord wound onto a capstan. For the experiments presented here, the stroke length for the pump was set to $L_{s}=70 \mathrm{~mm}$ and the stroke time held constant at $T_{S}=141.9 \pm 1.1 \mathrm{~ms}$. The formation number for the vortex rings,

$$
N=\frac{L}{D_{t}}=\frac{L_{s} D_{s}^{2}}{D_{t}^{3}}
$$

is around one. Here, $L$ is the length of the slug of water expelled from the tube. The Reynolds number, $R e=V_{r} D_{t} / v\left(V_{r}\right.$ is the vertical propagation velocity of the ring before the deceleration starts), is kept constant in all the experiments with a value around $5 \times 10^{3}$; some other researchers prefer to use the Reynolds number based on the circulation, $\operatorname{Re}_{\Gamma}=\Gamma / v$ (being $\Gamma$ the circulation), which in our case is a value in the order of $2 \times 10^{3}$. This value lies within the laminar regime and is comparable to the lower circulation Reynolds number cases of the experiments performed by other authors (i.e., Refs. 17-19), and is within the larger scenarios performed by other research articles (i.e., Refs. 11-13). 


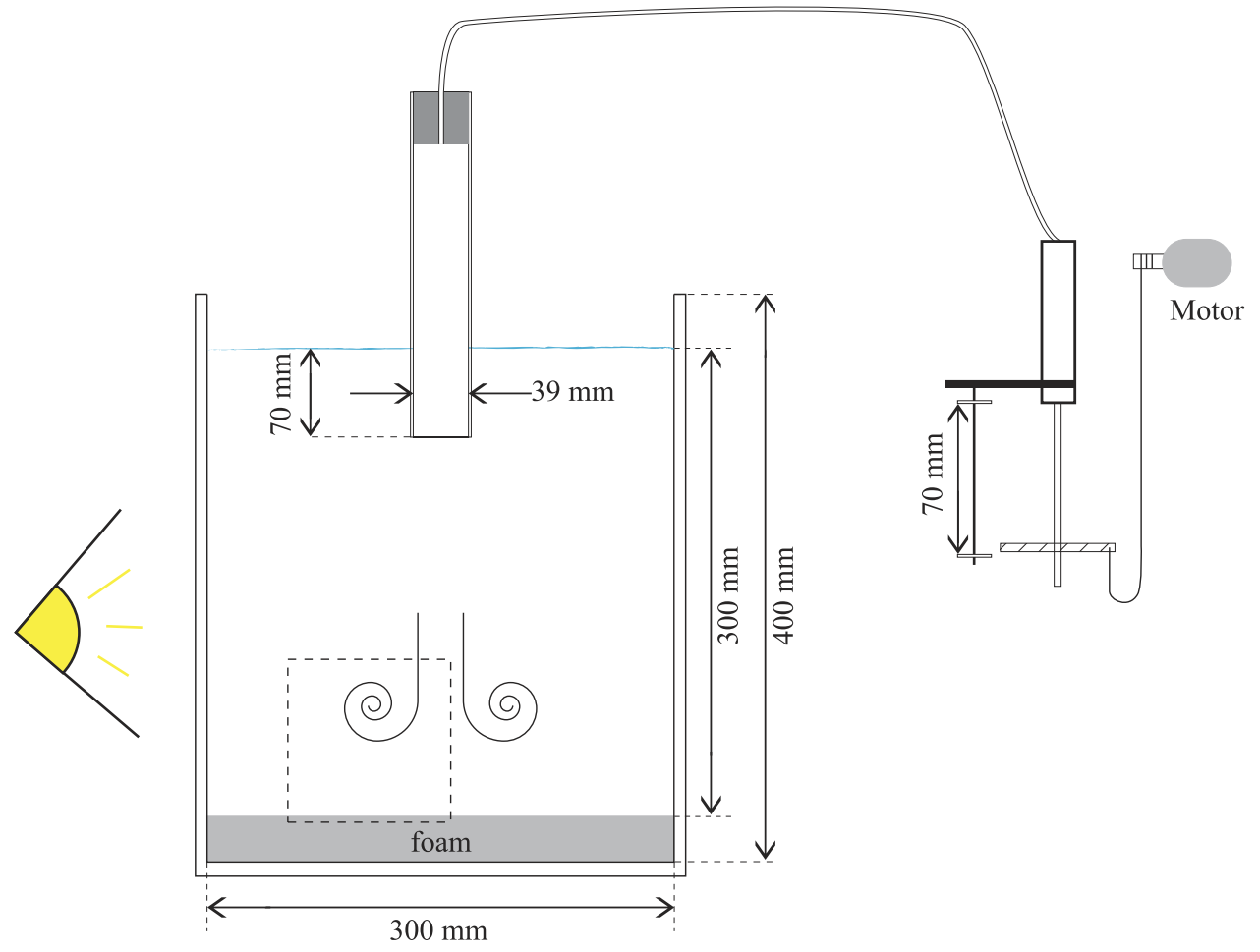

FIG. 1. Sketch of the experiment setup. Dashed square marks the field of view recorded.

Table I describes the main characteristics of the four different reticulated polyether foams used to form the porous boundary. Each had an internal structure that was geometrically similar but differed in scale (pore diameter), see Figure 2. The vertical permeability component of the permeability tensor of each foam,

$$
\boldsymbol{k}=\boldsymbol{K} \frac{v}{g}
$$

was determined by ensemble averaging the results obtained from 20 different Darcy's tests (UNE103403-99) for each foam with an estimate error of $\pm 2.5 \times 10^{-8} \mathrm{~m}^{2}$ for $k_{z}$. These data are given in Table I. For convenience, we identify the foam blocks based on the permeability values shown in Table I. Foam blocks k26, k51, and k70 all had a thickness of $h=25 \mathrm{~mm}$, while k85, the coarsest (most permeable) foam, was thicker with $h=50 \mathrm{~mm}$. We observed a weak anisotropy in the foams but calculations under the assumption of Darcy's flow showed the anisotropy had negligible impact on the flow. In all cases, we define our coordinate system so that $z=0$ is the top of the block of foam.

TABLE I. Characteristics of the foams used. Ppi range and height $(h)$ values are given by the manufacturers. Vertical hydraulic conductivity $\left(K_{z}\right)$ values are obtained experimentally and vertical permeabilities are obtained using viscosity at $20^{\circ} \mathrm{C}$. Pore diameter $\left(D_{p}\right)$ is obtained from visual observations.

\begin{tabular}{lccccc}
\hline \hline Foam name & $\begin{array}{c}\text { Ppi } \\
\text { range }\end{array}$ & $\begin{array}{c}D_{p} \\
(\mathrm{~mm})\end{array}$ & $\begin{array}{c}h \\
(\mathrm{~mm})\end{array}$ & $\begin{array}{c}K_{z} \\
(\mathrm{~m} / \mathrm{s})\end{array}$ & $\begin{array}{c}k_{z} \\
\left(10^{-8} \mathrm{~m}^{2}\right)\end{array}$ \\
\hline k26 & 60 & 0.5 & 25 & 0.24 & 26 \\
k51 & 30 & 1 & 25 & 0.48 & 51 \\
k70 & 20 & 2 & 25 & 0.65 & 70 \\
k85 & 10 & 3 & 50 & 0.79 & 85 \\
\hline \hline
\end{tabular}




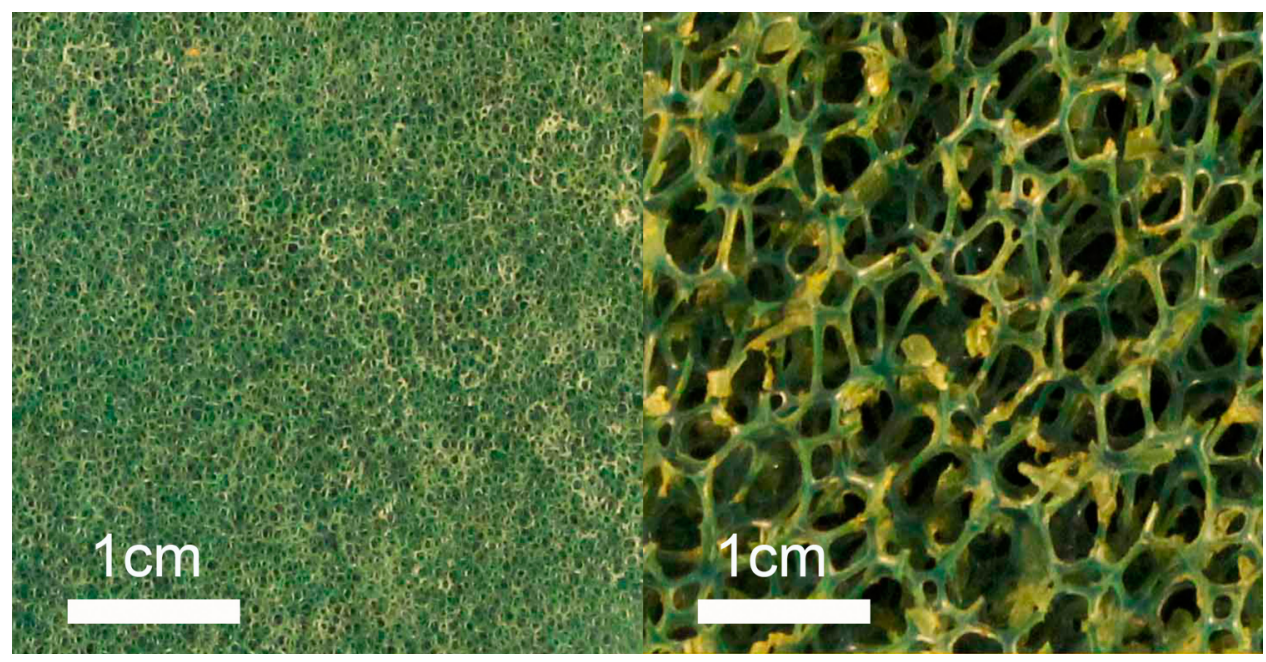

FIG. 2. Left: photo of the k26 foam. Right: photo of the k85 foam.

Before each experiment, care was taken to ensure that no air bubbles were caught in the foam (a small quantity of wetting agent was used to assist this process and the foam blocks were kept submerged between experiments).

In addition to the four porous foams, we studied the impact of the ring on a solid boundary, $\mathrm{k} 0$. We could treat these data as either the limit of zero permeability (placing our coordinate origin $z=0$ at the solid boundary) or the infinite permeability limit for a foam block of thickness $h$ by considering the bottom of the tank as $z=-h, k \infty$. The tank bottom was smooth. However, its classification with the porous foams was complicated by the existence of the flux of fluid and the horizontal momentum across the nominal upper surface. Therefore, in the present experiments, we are unable to detect any influence of the boundary roughness.

The experiments presented here were illuminated by a light sheet from a $300 \mathrm{~W}$ xenon arc lamp fitted with a parabolic dichroic reflector. Nearly, collimated light from the lamp passed between adjustable aluminium strips on the side of the tank to generate a sheet with a thickness of about $3 \mathrm{~mm}$. The experiments were recorded using a high-speed 1 MPixel camera (Photron SA1.1) at 1000 frames/s. The camera was fitted with a $60 \mathrm{~mm}$ AF micro NIKKOR lens with a $\mathrm{f}=2.8$ aperture. For some experiments, the field of view covered the whole diameter of the vortex ring, although for others, only one side of the ring was visualised in order to improve spatial resolution. For such experiments, the camera was located around $360 \mathrm{~mm}$ from the light sheet.

Our main experimental results were obtained using Particle Image Velocimetry (PIV) on one half of the vortex ring (see sketch in Figure 1). As discussed in Sec. III, our field of view was sufficient to ensure it captured the majority of the interaction between the ring and the porous boundary. We used Pliolite VTAC particles with nominal diameter between 70 and $110 \mu \mathrm{m}$ and specific gravity around 1.02. These particles were rendered neutrally buoyant through the addition of $35 \mathrm{~g} / \mathrm{l}$ of salt $(\mathrm{NaCl})$ to the water in the tank. The PIV analysis was performed using the software DigiFlow ${ }^{23}$ with interrogation regions $21 \times 21 \mathrm{px}^{2}$ at a spacing of 15 pixels giving an effective spatial resolution of $1.4 \mathrm{~mm}$. A cubic spline algorithm was used to interpolate between PIV results and acquire feasible results at every pixel, as part of an image distortion scheme used in the pattern matching process.

We also present experiments visualised using a precipitation technique driven by the electrolysis of electrical solder. A thin solder-covered ("tinned") copper foil was stuck to the inside of the open end of the PVC tube. A brief pulse of current was passed through this foil (attached to the positive side of a DC power supply) to produce a cloud of white precipitate just prior to ejecting the vortex ring (hydrogen bubbles were produced at the second electrode that was placed in a remote corner of the tank). This precipitate was largely confined to the boundary layer exiting the tube and so was wrapped up into the core of the vortex ring. Illuminating the whole domain allowed us to confirm that the rings remained essentially axisymmetric throughout their interaction with the porous boundary. 


\section{EXPERIMENTAL RESULTS}

We begin with some qualitative visualisations of the interaction between the vortex ring and the various boundaries using the precipitation technique described in Sec. II. Using a sheet of light passing through the axis of the ring, Figure 3(a) shows the interaction with a solid boundary, k0, while Figure 3(b) shows the interaction with the $\mathrm{k} 85$ (coarsest) foam. Both images are for the same time after generating the ring. In the absence of the boundaries, the two rings would be indistinguishable and their cores would be located at $z=0$, the position of the boundary. However, Figure 3(a) illustrates clearly the radial stretching of the ring as it begins to interact with its "image" in the solid boundary. In contrast, the concept of an image vortex ring to impose no normal flow across the boundary is not applicable to the porous boundary in Figure 3(b). Although there has been some stretching and deceleration of the ring, this is nowhere near as pronounced as was seen for the solid boundary, and consequently the core diameter remains large. As we shall see, this behaviour is typical for the permeable interactions. The ring's interaction with the solid boundary also deposits secondary vorticity of the opposite sign on the wall as a result of the no-slip boundary condition. The presence of a small amount of precipitate outside the core of the ring makes this visible in Figure 3(a), where separation of this secondary vorticity is leading to the emergence of a coherent secondary vortex that is beginning to wrap some of the precipitate around it. While this is happening around the entire body of the vortex ring, the illumination makes this clearer in the vicinity of the left-hand core in Figure 3(a). In contrast, there is no clear evidence from Figure 3(b) of such a structure existing in the interaction with the porous $\mathrm{k} 85$ boundary.

Figure 4 offers the same form of visualisation across our entire range of porous and solid boundaries. These images are arranged so that the boundary permeability increases from left to right. The upper row of the figure (Figures 4(a)-4(e)) shows the similarity of the rings at a height $z=D_{t}$ above the wall (henceforth we label this height as our time origin $t=0$ ). The cores of the rings are at the same height and of the same size; small variations in the $\mathrm{k} 0$ case are due to imprecisions in the way the precipitate is introduced and henceforth considered negligible not only in the k0 case but also in other cases. The images in the lower row of Figure 4 (panels (f)-(j)) are from the same five experiments as the upper row but show the position of the cores with $\tilde{t}=\frac{V_{r}}{D_{t}} t=1.05$. Clearly, increasing the
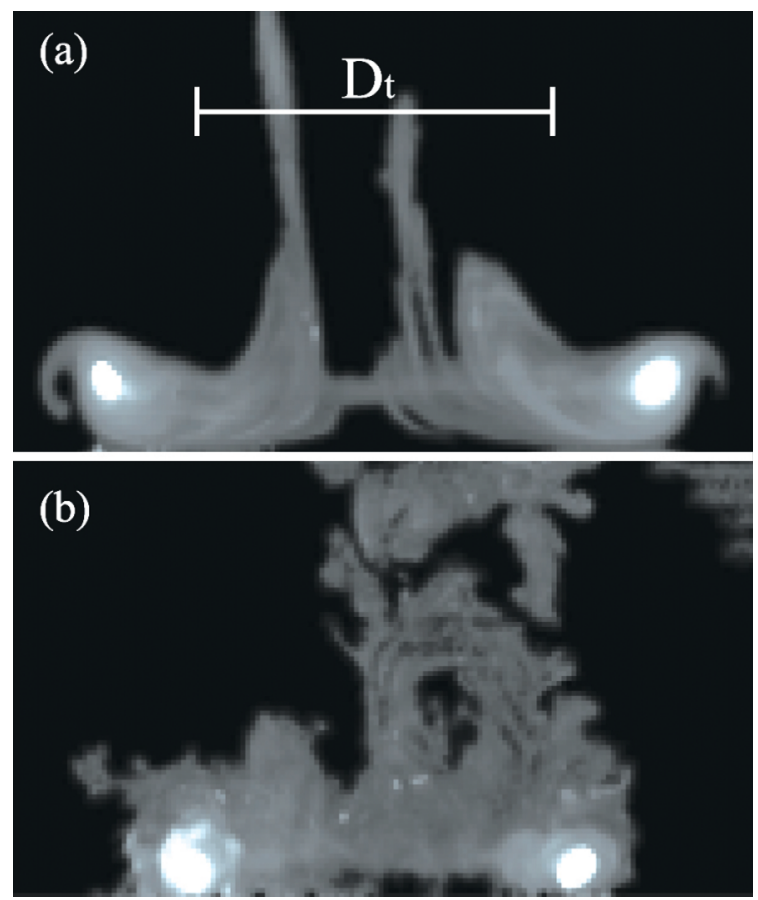

FIG. 3. Comparison of the vortex ring interaction between solid boundary—upper-and a coarse foam—lower-visualized using the electrolytic precipitation of tin chloride. 


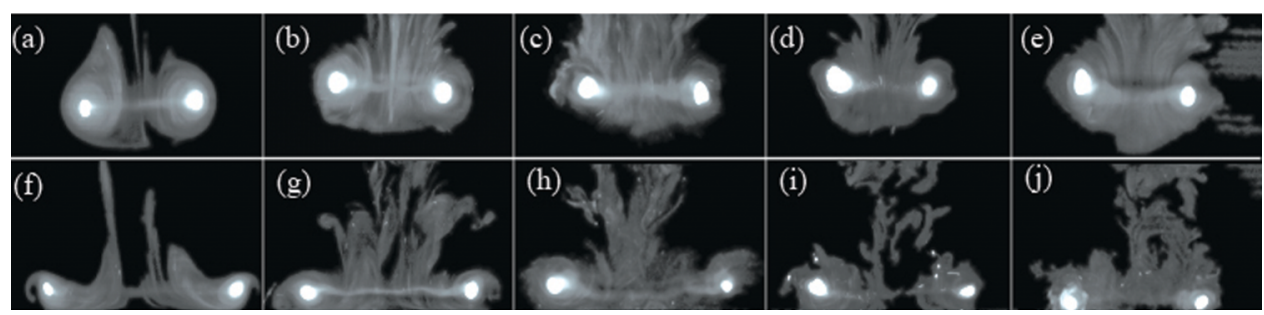

FIG. 4. Upper row $\tilde{t}=0$; lower row $\tilde{t}=1.05$. Column-wise, from left to right with increasing permeability k0 ((a) and (f)), k26 ((b) and (g)), k51 ((c) and (h)), k70 ((d) and (i)), k85 ((e) and (j)).

permeability allows the rings to approach the boundary more closely while reducing the stretching of the diameter of the ring. The precipitate outside the core may give the appearance to have tilted vortex rings. However, this effect is due to the Kelvin waves, or azimuthal instabilities, meaning the slice through the ring may sample the core at different phases producing this apparent inclination.

The PIV experiments give more detail about the different behaviour when using porous boundaries. Figure 5 shows velocity and vorticity fields at different dimensionless times for the $\mathrm{k} 0$ and k85 boundaries. Figures 5(a)-5(c) show the flow above the k0 solid boundary (the symmetry axis of the vortex ring is located on the left-hand side of the field of view). As seen by previous authors and noted above, the no-slip boundary condition has generated secondary vorticity at the boundary that has begun to separate to form a secondary vortex ring. This secondary vortex ring interacts with the primary ring to further retard and temporarily reverses the primary ring's direction of vertical propagation. Secondary vorticity continues to be generated at the boundary and is wrapped around the primary ring as the stronger circulation in the primary ring sweeps the secondary ring out and around it before compressing it back towards the axis. As discussed by others (e.g., Refs. 8 and 24), the compression of this secondary ring plays an important role in the development of instabilities and the eventual break-up of the primary vortex ring.

Figures 5(d)-5(f) show how the coarsest foam (k85) fundamentally changes the nature of the interaction. First, the k85 boundary lets the vortex ring get closer to the boundary and the core begin

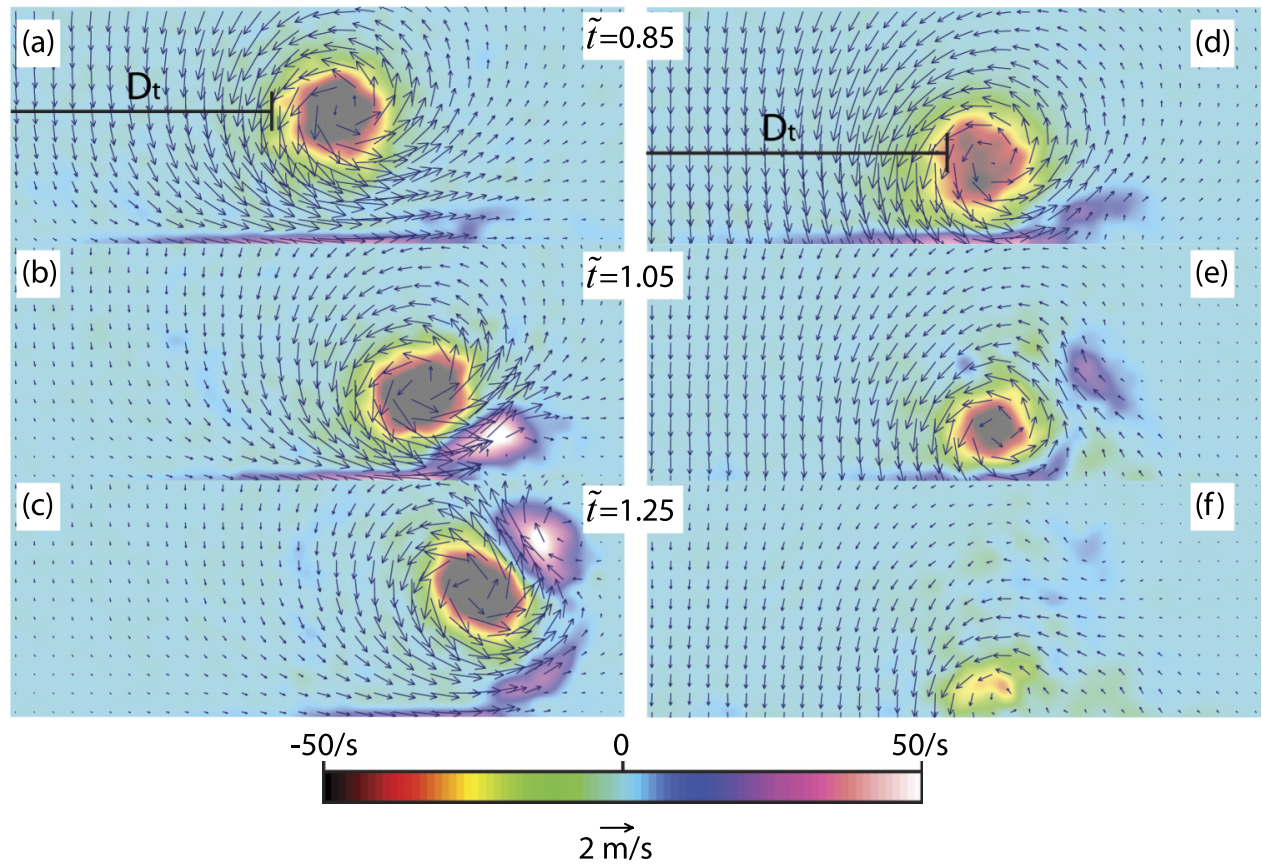

FIG. 5. PIV results of a vortex ring approaching two different boundaries: (a)-(c) solid boundary (k0); (d)-(f) coarsest foam k85. Background variable: vorticity. 
to penetrate it. Second, the changes in diameter are not as significant when a permeable boundary is used since the secondary vortex does not have the same intensity as in the case of the solid boundary interaction. Finally, although secondary vorticity is perceptible in Figures 5(d) and 5(e), it is comparably weaker than the solid boundary case, indicating it can also affect the relevance of the no slip boundary condition assumed in the $\mathrm{k} 0$ scenario.

In Figure 6, we summarise the behaviour of the core of the primary vortex ring with the ensemble of 10 PIV experiments for each of the different boundary permeabilities. In particular, we use the vorticity criterion of Bethke and Dalziel ${ }^{19}$ to locate the cores of the vortex rings from the PIV measurements. The trajectory of the cores is shown in Figure 6(a). Here, we plot $\tilde{Z}=\frac{Z}{D_{t}}$ against $\tilde{D}=\frac{2 R}{D_{t}}$, where $R$ is the distance from the symmetry axis to the centre of the core and $Z$ represents the vertical position of the centre of the core. In the absence of a lower boundary, the trajectory would be a vertical line with constant $\tilde{D}$. The solid line shows the behaviour of the core above the solid boundary, the diameter increasing as the ring approaches the boundary. Note the characteristic rebound of the core at $\tilde{D} \approx 1.7$. This is due to the coupling between the primary ring and the secondary ring following separation of the boundary layer. Figures $6(\mathrm{~b})$ and $6(\mathrm{c})$ show the same trajectory data plotted as a function of dimensionless time. For the $\mathrm{k} 0$ boundary, the rebound is clearly visible after the ring's closest approach at $\tilde{t} \approx 1$ with $\tilde{Z}$ increasing then $\tilde{D}$ decreasing from $\tilde{t} \approx 1.1$.

As the permeability of the boundary increases (k26, dotted-dashed lines), the diameter of the ring grows slightly more slowly (Figure 6(c)) and the vertical velocity is reduced by less as it approaches the boundary (Figure 6(b)) resulting in the trajectory lying below that of the solid boundary until around $\tilde{t}=1$, when the ring decelerates relatively quickly. A small rebound is evident, although compared with the rebound from the solid boundary, the rebound from k26 is smaller, earlier, and at smaller radius. The trajectory above the $\mathrm{k} 51$ porous boundary (dotted line) shows a slightly closer initial approach, more sudden and slightly later deceleration, and a smaller spread than either the solid boundary or k26. Although the approach of the core still changes direction (with the core moving away from the boundary for $\tilde{t}>1.0$ ), the diameter grows monotonically until much later.

The trajectories above the two coarsest foams (k70 dotted-dotted-dashed lines and k85 long dashes) continue the trend of not expanding as much as they approach the boundary. Their approach velocity remains constant until about $\tilde{t}=0.8$, after which they decelerate and begin to grow in diameter more dramatically. There is some suggestion of a weak rebound for the k70 boundary (although the distance from the boundary remains nearly constant after $\tilde{t} \approx 1$, the diameter decreases slightly), but none for the most permeable boundary, $\mathrm{k} 85$.

One open question is whether the thickness of the porous layer plays a role. It is obvious that for the same vortex ring characteristics impacting a very thin porous layer, the thickness will be important (comparing two foams with the same permeability), but it is less clear whether our current porous layers are sufficiently thick for their thickness to be unimportant, considering the tank has an impermeable base. To this end, the grey line in Figure 6 replots the trajectory for the solid boundary case but offset downwards by $h=50 \mathrm{~mm}$, the thickness of the most permeable (k85) foam. We can view this as representing the limit of high permeability (with $h=50 \mathrm{~mm}$ layer thickness) where the porous boundary ceases to play a significant role and only the solid boundary of the tank is important. As can be seen in Figure 6(a), the trajectory above this virtual $\mathrm{k} \infty$ foam by the time the ring reaches $\tilde{Z}=0$ is nearly uninfluenced by the presence of a boundary and is clearly different from the ring approaching the $\mathrm{k} 85$ foam.

According to Bethke and Dalziel, ${ }^{19}$ a vortex ring impinging a solid boundary begins to stretch its diameter and decelerates at a height comparable to $D_{t}$. Figure 7(a) quantifies the height at which the diameter of the ring starts increasing detected in Figure 6(c), $Z_{t}$. As seen in Figure 7(a), the vortex ring velocity remains constant for longer (to a lower height) with more permeable boundaries. The extreme case is the $\mathrm{k} 85$ foam, when the ring begins to decrease its downward propagation velocity at a height equal to only $20 \%$ of the diameter of the tube. On the other hand, Figure 7(b) plots the maximum rebound height, $Z_{r}$, the maximum height of the primary core after $\tilde{t}>1$ in Figure $6(\mathrm{~b})$. In the most permeable case, $k 85$, no rebound has been observed; we flag this by setting $Z_{r}=0$. Smaller permeabilities allow the vortex ring to slightly rebound, increasing $Z_{r}$ up until its maximum in the solid boundary case when it is around a quarter of the tube diameter, $D_{t}$. 

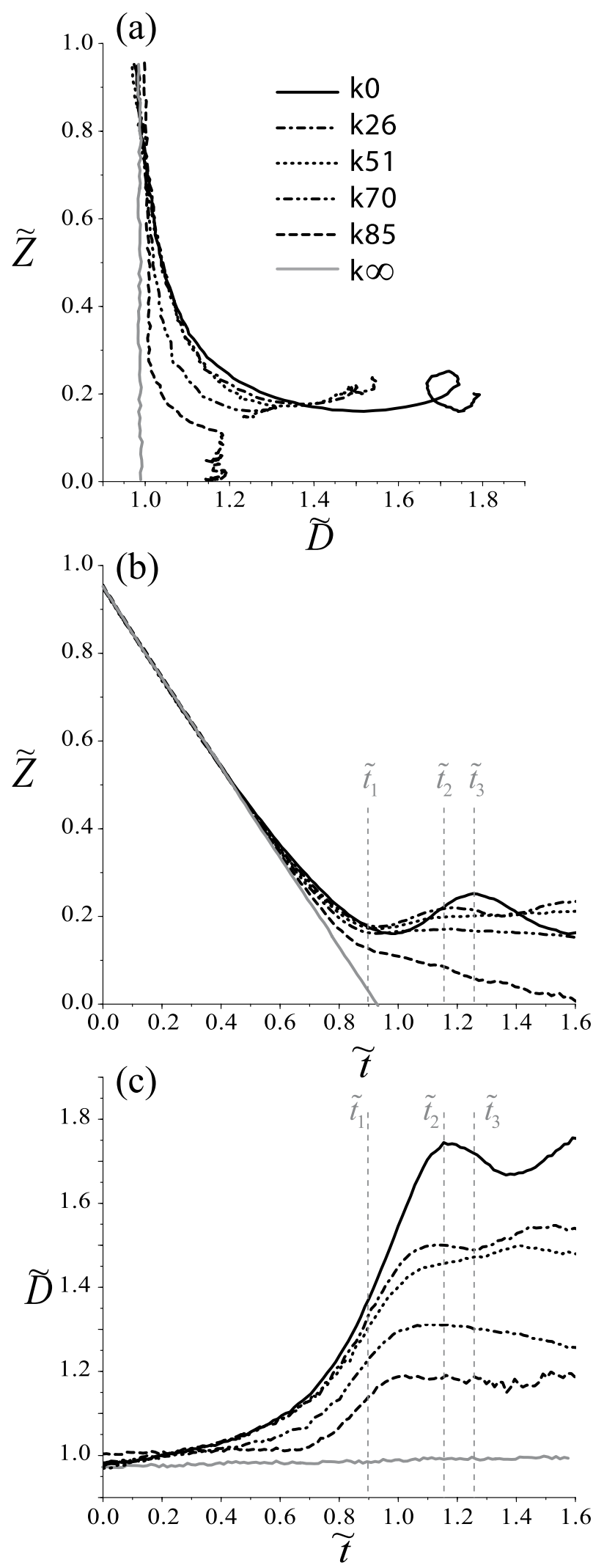

FIG. 6. Comparison between boundary types with porous boundaries and solid boundary. (a)Trajectory; (b) vertical position evolution; (c) diameter evolution. 

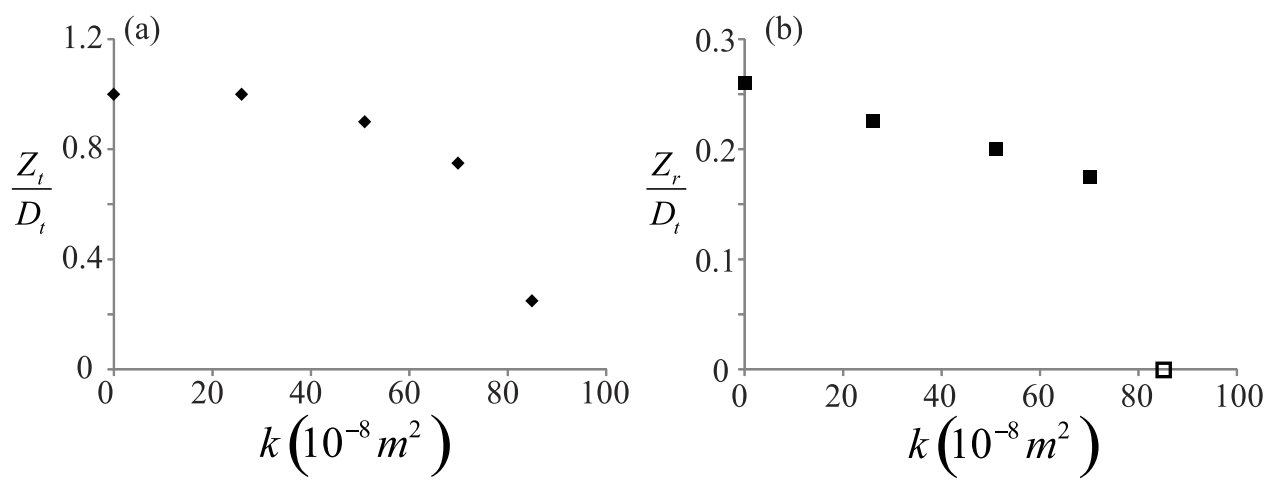

FIG. 7. Characteristic heights related to (a) the $\mathrm{Z}$ level at which the diameter of the initial ring started stretching and (b) the maximum $\mathrm{Z}$ level reached during the primary vortex rebound.

In order to reconcile the differences in behaviour of the vortex ring-boundary interaction, we examine the velocity and vorticity fields for each case in Figure 8. We use three specific times to compare all the different boundary types (see Figures 6(b) and 6(c)). Although the time for closest approach varies slightly with permeability (see Figures $6(\mathrm{~b})$ and $6(\mathrm{c})$ ), we shall take $\tilde{t}_{1} \approx 0.90$ as representative of this. Similarly, $\tilde{t}_{2} \approx 1.15$ marks the time at which the diameter is maximum for the $\mathrm{k} 0$ boundary, and $\tilde{t}_{3} \approx 1.25$ is the time of the maximum rebound height in the k0 boundary. Figure 8 shows how the secondary vortex ring is formed when the primary vortex ring interacts with a solid boundary. As has already been described, the secondary ring is formed with the detachment of the boundary layer and causes the decrease on the diameter of the primary vortex ring. From Figure 6(c), all boundary types except $\mathrm{k} 85$ presented a decrease in diameter indicating the formation of a secondary vortex ring. However, Figure $8(\mathrm{e})$ shows evidence of weak secondary vorticity in the k85 scenario, which may indicate why the primary ring in this case increases its diameter up to a certain point when a secondary vortex ring is formed (around $t \approx 1.0$ ). As described before, the formation of

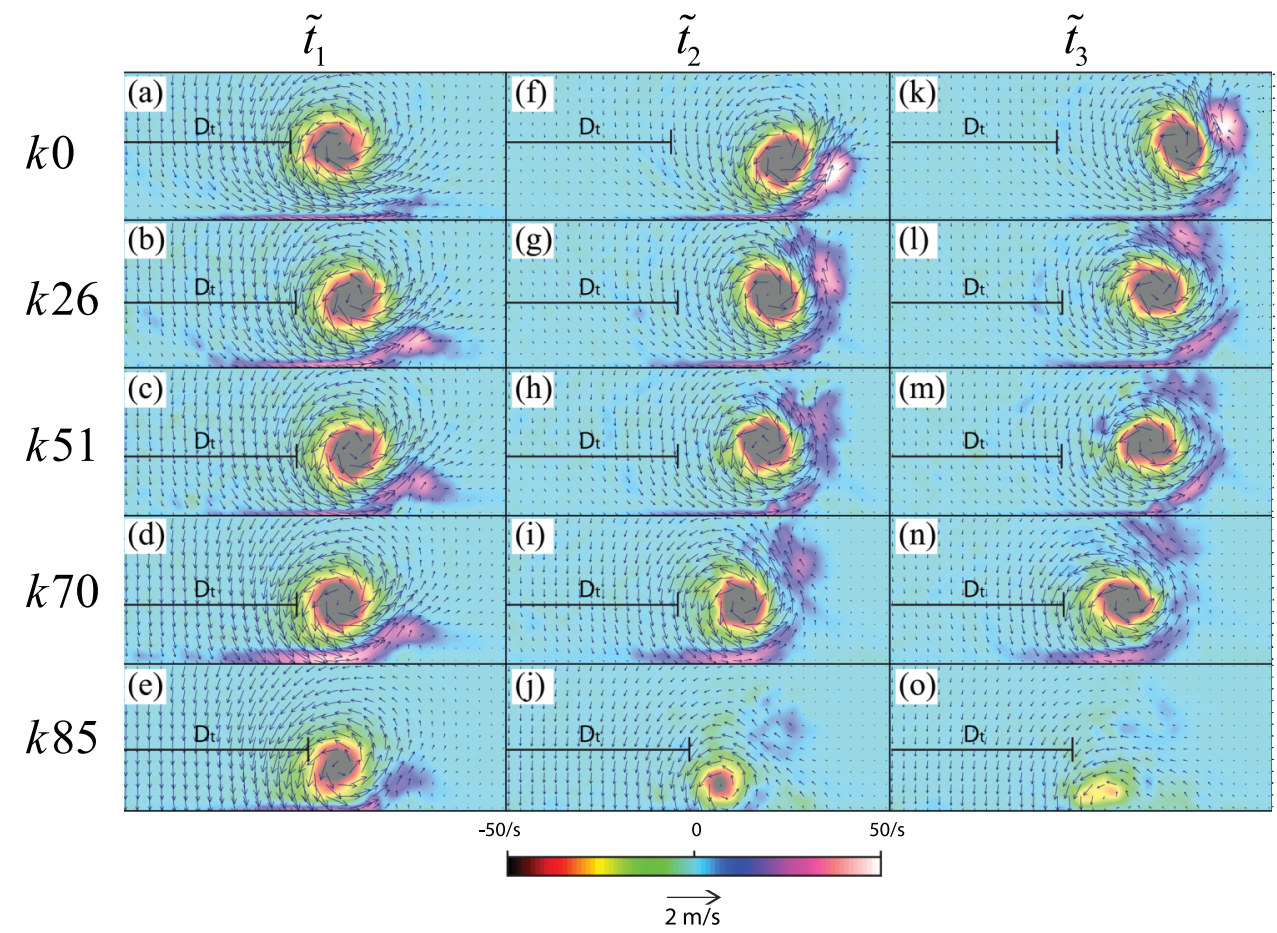

FIG. 8. PIV results of half vortex ring at the time steps $\tilde{t}_{1} \approx 0.90, \tilde{t}_{2} \approx 1.15, \tilde{t}_{3} \approx 1.25$. Background variable vorticity. 
this secondary ring is due to the development of the boundary layer. Beavers and Joseph, ${ }^{25}$ Taylor, ${ }^{26}$ and Richardson ${ }^{27}$ suggest that the boundary layer penetrates into the porous media. Hence, the weak formation of the secondary vortex ring is clearly explained by increment in the extension onto the foam material with porosity, affecting the boundary layer. This makes the detachment of the boundary layer more difficult and consequently inhibits the formation of this secondary vortex ring.

Figure $8(\mathrm{f})$ is useful to see how the maximum diameter of the primary ring is reached when the secondary vortex ring is at the same elevation as the primary vortex; Figures 8(g)-8(i) may confirm this since $\tilde{\mathfrak{t}}_{2}$ in the $\mathrm{k} 26, \mathrm{k} 51$, and $\mathrm{k} 70$ cases is soon after the maximum diameter is attained, see Figure 6(c), and the secondary vortex ring is located slightly above the primary ring. The coarsest boundary, $\mathrm{k} 85$, does not show the complete evolution of the secondary vorticity because the ring seems to penetrate into the foam. Finally, Naaktgeboren et al. ${ }^{21}$ described the existence of a third weak vortex ring coming from the boundary layer which is observed in all foams except the $\mathrm{k} 85$ boundary in Figures $8(\mathrm{k})-8(\mathrm{n})$.

One variable of interest to emphasize the changes on the vortex ring interaction with different permeable boundaries is the evolution of the vortex ring circulation,

$$
\Gamma=\int_{A_{c}} \omega d A
$$

where $A_{c}$ is the area of the core. As detailed in Bethke, ${ }^{16}$ the definition of the core is somewhat controversial. Here, vorticity lying below $3 \%$ of the vorticity peak is considered as noise and not used in the computation. Maximum vorticity (here negative) is found at the centre of the ring's core. Therefore, primary circulation is computed as sum of the negative values within the defined threshold and the secondary circulation as the sum of positive values above the absolute value of the same threshold. This is accurate for the primary vortex ring but may underestimate the circulation of secondary vortex ring and boundary layer (where a larger fraction of the vorticity may be excluded from the circulation calculation). However, this methodology is sufficient to reveal the evolution of the circulation of the secondary vortex, once the primary ring has started its rebound and results are not sensitive to small changes in the threshold of the 3\%. During the vortex ring's approach to the wall, the secondary vorticity is generated in the boundary layer; after the vortex ring has reached the wall, and at the early stages of the secondary vortex formation, secondary vorticity from the boundary layer still represents the majority of the secondary circulation. However, for $\tilde{t}>1$, the secondary vortex has formed through separation of the boundary layer and this secondary vortex represents the dominant contribution to the secondary circulation.

Figure 9 plots the measurements of the non-dimensional circulation, $\tilde{\Gamma}=|\Gamma| /\left(V_{r} D_{t}\right)$, for both the primary and secondary vorticities. The lower values of $\tilde{\Gamma}$ in the $\mathrm{k} 85$ case of primary circulation (Figure 9(a)) are mainly due to small errors accumulated on the computation of the vertical velocity propagation of the ring before the beginning of the deceleration, $V_{r}$. When the ring is impinging a solid boundary, secondary vorticity appears due to the viscosity and the no slip boundary condition. Hence, secondary circulation starts increasing while the primary vortex ring approaches the wall because the boundary layer at the wall starts developing; this is why the secondary circulation starts increasing before the decrease in primary circulation. Primary circulation of the ring is preserved during the initial stretching. However, when the ring is closer to the wall $\left(\tilde{t}=\tilde{t}_{1}\right)$, both the primary and secondary vorticities interact through molecular viscosity. From this time on, primary circulation decreases while the secondary ring is being formed by the detachment of the secondary vorticity present in the boundary layer. In the k0 scenario, circulation of the secondary vorticity has its peak coinciding with the point where ring reaches its maximum diameter. After $\tilde{t}_{1}$, the primary circulation decrease is faster for higher permeable boundaries, indicating that the interaction between the primary and secondary vortex ring is generating more losses.

Figure 9(b) does not show a clear pattern of relationship between the secondary circulation and permeability. This is caused by the strong influence of the interface level $\tilde{Z}=0$ on the curves, mainly due to the light reflection contaminating the results. Around $\tilde{t}_{1}$, we can distinguish two different behaviours: (i) for the solid boundary case, $\mathrm{k} 0$, secondary circulation increases faster after $\tilde{t}_{1}$, indicating that the boundary layer keeps forming at the interface; (ii) for all the permeable boundaries, secondary circulation peaks either keeps constant for the lower permeable cases, k26 and k51, or decreases for 
a)

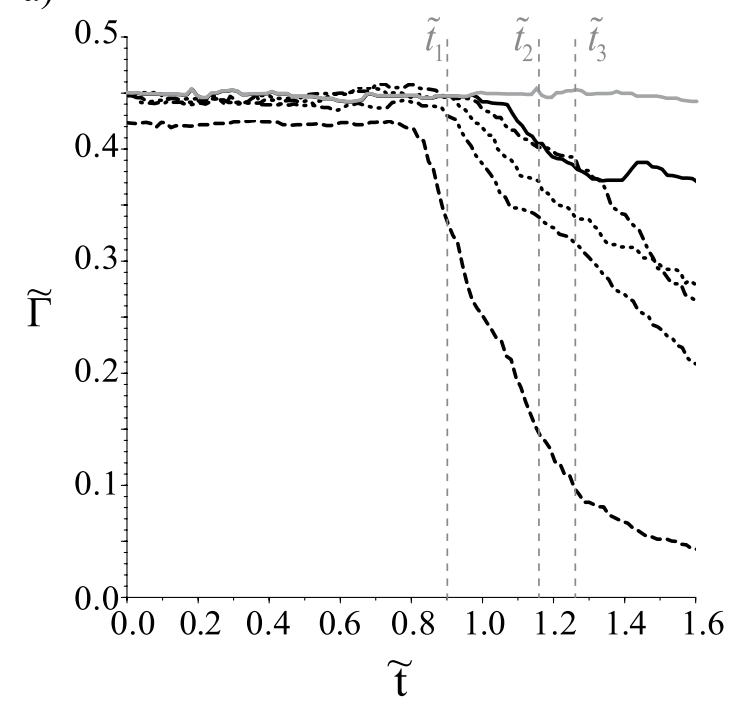

b)

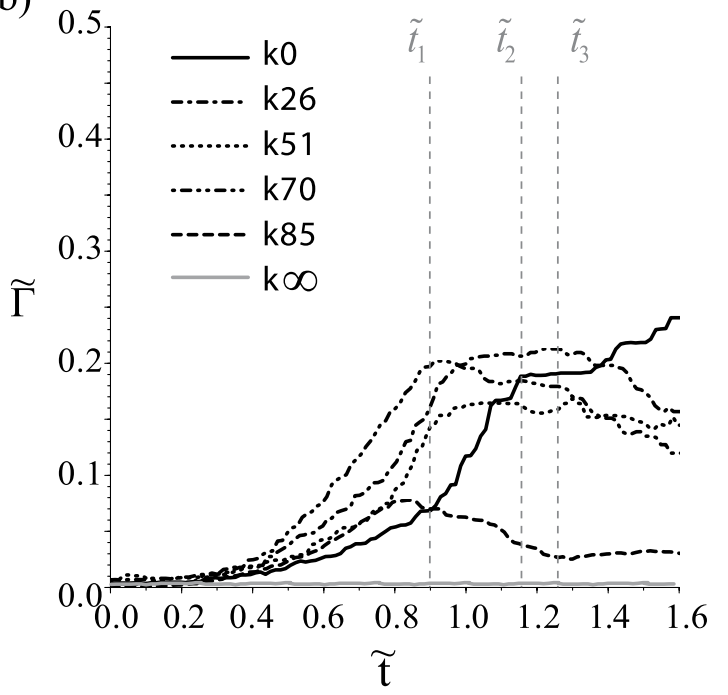

FIG. 9. Evolution of the primary (a) and secondary (b) vorticity of the interaction of a vortex ring with different boundaries.

the larger permeable cases, $\mathrm{k} 70$ and $\mathrm{k} 85$. Therefore, the boundary layer at the interface does not grow as it does for the solid boundary case affecting the secondary vortex ring formation and life: secondary vortex ring is weaker as the permeability increases, as shown in Figure 8. Finally, Figure 9(b) reflects the existence of secondary vorticity in the coarsest permeable boundary, $\mathrm{k} 85$, which can be related to the formation of the secondary vortex ring as was detected in Figure 8(e).

Figure 10 shows the vertical (left panel) and the horizontal (right panel) dimensionless velocity profiles $\left(\tilde{v}=v / V_{r}\right)$ measured $1 \mathrm{~mm}$ above the permeable or solid boundary. The radius has been made dimensionless by $\tilde{R}=R / D_{t}$. The marks represent the radial position of the core at each time, and the time profiles coincide with the frames plotted in Figure 8. In absolute terms, vertical velocities close to the boundary increase with permeability whereas horizontal velocities decrease. Larger horizontal and vertical velocities are reached at $\tilde{t}_{1}$, except for the $\mathrm{k} 0$ and the $\mathrm{k} 85$ cases, compared to other instants in the figure. In the $\mathrm{k} 0$ scenario, this is because the ring has not reached its closest approach to the boundary, whereas in the most permeable $\mathrm{k} 85$ foam, the maximum velocities are obtained at the inflectional point in the curve showed at Figure 6(b). Regarding the position of the core with respect to velocity peaks, two behaviours are observed in Figure 10. First, the core is located between positive and negative vertical velocity peaks, but always closer to the positive peak. Alongside this, the vertical 


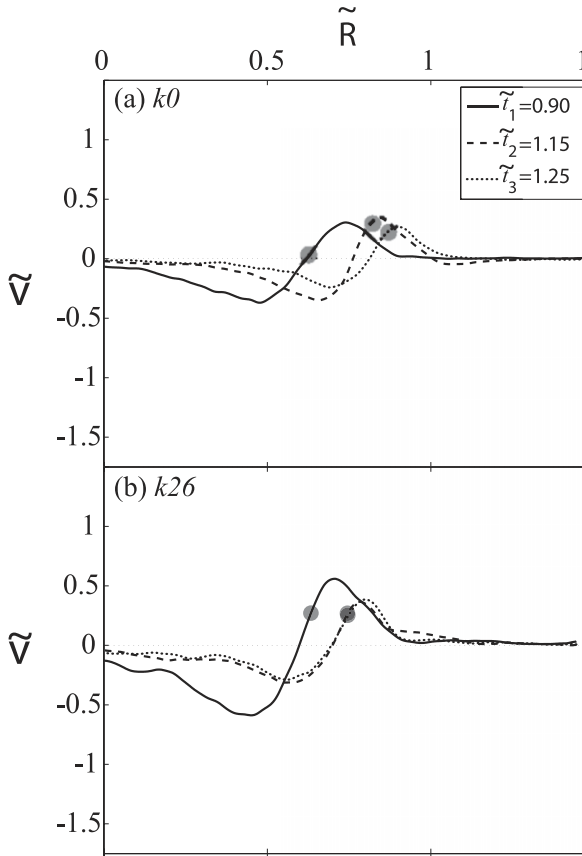

(c) $k 51$

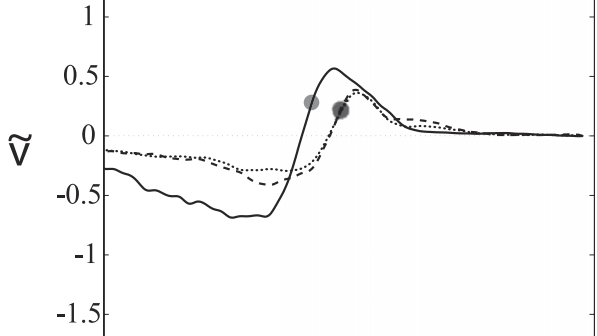

(d) $k 70$

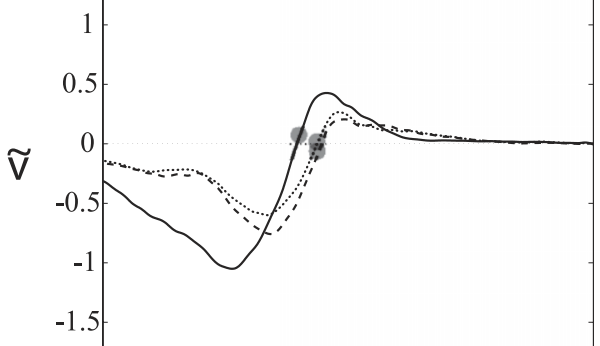

(e) $k 85$

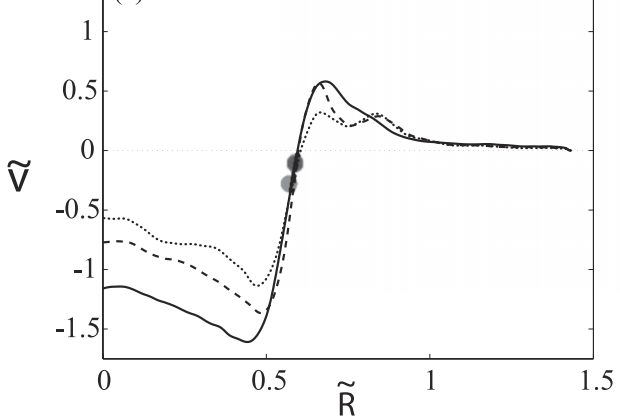

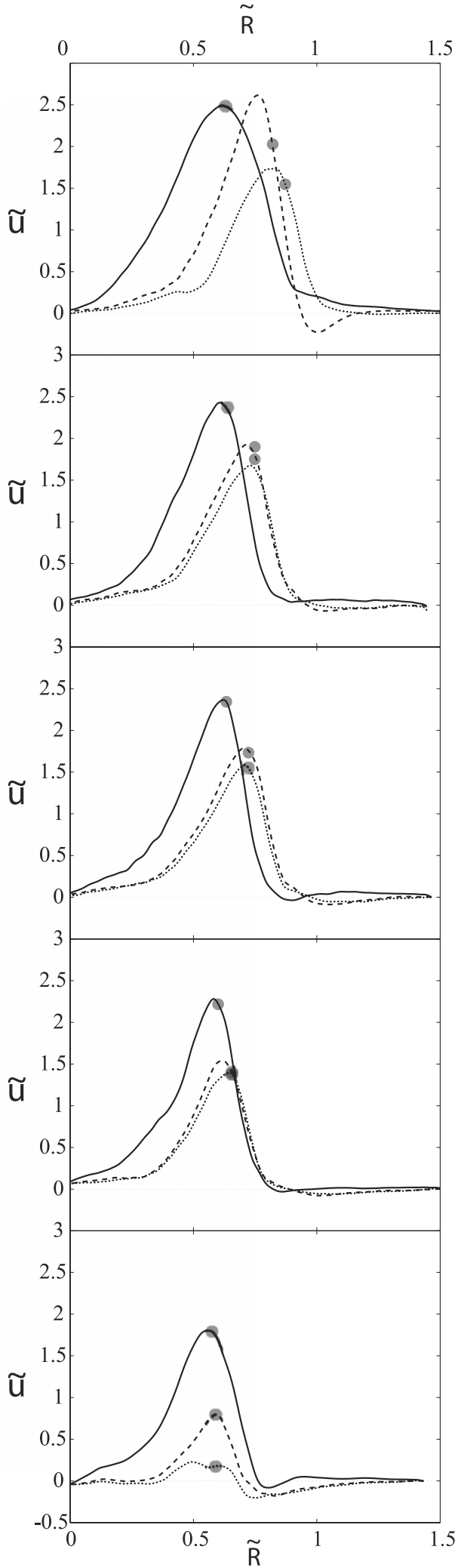

FIG. 10. Left column: vertical velocity profiles; right column: horizontal velocity profiles. Results obtained from the PIV velocity fields $1 \mathrm{~mm}$ above the boundary limit at the same instants as Figure 8. Marks define the position of the centre of the core at each time. 


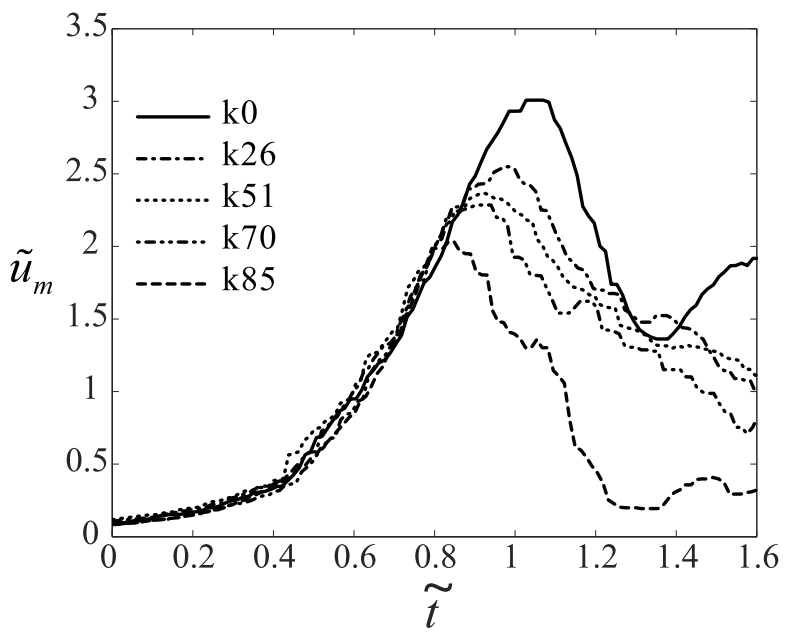

FIG. 11. Non dimensional maximum horizontal bed velocity evolution on dimensionless time.

velocities below the core are generally positive and have an influence on slowing down the core, with the exception of the most permeable $\mathrm{k} 85$ foam, where the velocity in the bed located right below the core is negative. The second behaviour detected in the right panel of Figure 10 is that, in contrast with the vertical velocity profiles, the bed horizontal velocity peak is located slightly closer to the axis of symmetry than the core of the primary ring, particularly for the $\mathrm{k} 0$ solid boundary. This is caused by the no-slip boundary condition present in the solid boundary case, and will be further discussed for the permeable cases by comparing the evolution of the horizontal velocity peaks.

Figure 11 plots the peak horizontal velocity as a function of time, following, ${ }^{19}$ specifically

$$
\tilde{u}_{m}(t)=\max _{r} \tilde{u}(z, r, t)
$$

with the criteria of bed velocity defined at a height $\mathrm{z}=1 \mathrm{~mm}$, used throughout the present article. Bethke and Dalziel ${ }^{19}$ found a clear deviation of the solid boundary curve from the inviscid theoretical curve. Moreover, they reported that the same curve for a sediment bed layer did match perfectly with the inviscid plot suggesting that this latter scenario presented a macroscopic free-slip boundary condition (at least in the neighbourhood of the maximum). However, in Figure 11, permeable boundaries do not differ from the solid boundary curve mainly because the measurements are made $1 \mathrm{~mm}$ above the bed whereas ${ }^{19}$ measured at $0.5 \mathrm{~mm}$. Assuming the diffusion of vorticity over a time as $D / V_{r}$, then a good approximation to the boundary layer thickness in the $\mathrm{k} 0$ case is $\delta \approx\left(v D_{t} / V_{r}\right)^{1 / 2}$ which yields to a $0.5 \mathrm{~mm}$ value, suggesting the velocities at $z=1.0 \mathrm{~mm}$ will be largely uninfluenced by the no-slip condition. The use of $z=0.5 \mathrm{~mm}$, which may have provided greater insight into the macroscopic boundary condition, was not feasible due to the nature of the foam.

In Figure 11, time evolution of this maximum horizontal bed velocity is the same for all experiments at early stages of the vortex ring motion. However, after bed velocity for the coarsest boundary, $\mathrm{k} 85$, reaches its maximum $\tilde{u}_{m}$ at $\tilde{t} \approx 0.85$, it decreases and diverges from the general trend of the curve. Similarly, lower permeability boundaries experience the same phenomenon at the times detailed in Table II. This peak takes place later when permeability decreases-and with higher horizontal velocities - and coincides approximately with the time at which the ring reaches its minimum

TABLE II. Time occurrence when maximum horizontal bed velocity reaches its peak.

\begin{tabular}{llllll}
\hline \hline & $\mathrm{k} 0$ & $\mathrm{k} 26$ & $\mathrm{k} 51$ & $\mathrm{k} 70$ & $\mathrm{k} 85$ \\
\hline$\tilde{t}$ & 1.07 & 0.96 & 0.91 & 0.89 & 0.85 \\
$\tilde{u}_{m}(\max )$ & 3.0 & 2.6 & 2.4 & 2.3 & 2.1 \\
\hline \hline
\end{tabular}


height and secondary vorticity starts detaching from the boundary. In the $\mathrm{k} 85$ case, however, since there is no minimum height, the peak coincides in time at which the slope of the $\mathrm{k} 85$ curve in Figure 6(b) changes. The important role played by the permeability differences can also be associated with the existence of fluid exchange between the ambient and the porous boundary. Since the measurements are $2 \mathrm{D}$, the total flux exchanged is computed in the observed area, on the basis that this is representative of all the volume under the assumption of axisymmetry.

To calculate fluxes, we should use the bed velocity at $z=0 \mathrm{~mm}$. However, this is not possible experimentally. In a similar calculation, Bethke and Dalziel ${ }^{19}$ used the velocities at $z=0.5 \mathrm{~mm}$ above a bed of particles, but here, we were only able to determine reasonable velocities down to $z=1 \mathrm{~mm}$. Consequently, we shall use the velocity at this height for our calculations.

The positive flux, $q^{+}$, is defined as the flow coming out of the foam $\left(v^{+}=v\right.$, when $\left.v>0\right)$ as

$$
q^{+}(t)=\Delta r \sum_{R_{i}} v\left(z_{0}, r_{i}, t\right) r_{i} \quad \forall r_{i}, v>0 .
$$

Similarly, the negative flux - ambient fluid moving into the porous boundary-is defined using the same terms for negative velocity points $\left(v^{-}=v\right.$, when $\left.v<0\right)$ as

$$
q^{-}(t)=\Delta r \sum_{r_{i}} v\left(z_{0}, r_{i}, t\right) r_{i} \quad \forall r_{i}, v<0 .
$$

Therefore, the net flux exchanged is the sum of Eqs. (5) and (6),

$$
q(t)=q^{+}(t)+q^{-}(t) .
$$

Assuming there is no flux exchange beyond the limits of the field of view, and that the foam may be considered rigid, we expect no net exchange between the foam and ambient fluid, and thus anticipate $q(t)=0$.

Figure 12 presents the positive and net flux exchange, both in dimensionless form, $\tilde{q}=q /\left(\pi D_{t}^{2} V_{r}\right)$. Figure 12(a) shows the positive flux calculated using Eq. (5) and Figure 12(b) plots the net flux computed using Eq. (7). The net flux for the solid boundary k0 was also computed to determine an estimate of the inherent error in the PIV interrogation process. In general, the net flux computed for the $\mathrm{k} 0$ case, Figure 12(b), is negative when the ring is approaching the boundary and starts oscillating. Therefore, the dimensionless mean estimative error, using the $\mathrm{k} 0$ results of net flux as a reference, is in the order of $10^{-4}$, two orders of magnitude below the maximum value of $q^{+}(t)$ observed for the porous boundaries. The positive flux, $q^{+}(t)$, in all boundary types is maximum when the primary vortex ring is at its closest approach from the boundary (except for $\mathrm{k} 85$ where it occurs when the ring clearly decelerates). However, the peak of the net flux (Figure 12(b)) is slightly retarded with the peak of the positive flux (Figure 12(a)), occurring when the ring diameter is the greatest. In Figure 12(b), two different behaviours are detected: (i) for the coarsest foams, $\mathrm{k} 85$ and $\mathrm{k} 70$, the total flux exchanged is mostly negative, whereas (ii) for the finest foams, $\mathrm{k} 51$ and $\mathrm{k} 26$, the total flux is smaller and positive particularly in k26. Predominantly, in Figure 12, conservation of mass $($ Eq. $(7)=0)$ is not satisfied at any time for any of the experimental measurements with the error always exceeding that for the $\mathrm{k} 0$ case.

There are four candidate mechanisms for the error in $q(t)=0$ : (i) the flow exiting the foam is three-dimensional with azimuthal variations not captured by the current methods; (ii) the relatively slow camera shutter speed means slower particles create brighter images near the bottom and the PIV may be biased towards them; (iii) the foam filters some of the particles from the flow so that there are many fewer particles in the upward flow (contributing to $q^{+}$) creating a bias in the measurements; and (iv) the limited resolution of the PIV processing that effectively smooths any localised fast-moving jets issuing from the individual pores. The first of these possibilities affects mainly the coarser foams due to higher velocities and larger pore diameter, introducing a larger 3D effect. The second candidate might explain the effects found on the solid boundary and the finer foams, where net flux is positive during all the experiments. The third candidate affects mainly coarser foams because incoming velocities (which are higher in the most permeable boundaries) make the particles lying at the surface of the foam be exhausted sooner. Moreover, this fluid may have been in the foam for a while and so is likely to have deposited its particles. Finally, the fourth candidate affects all foams independently. 

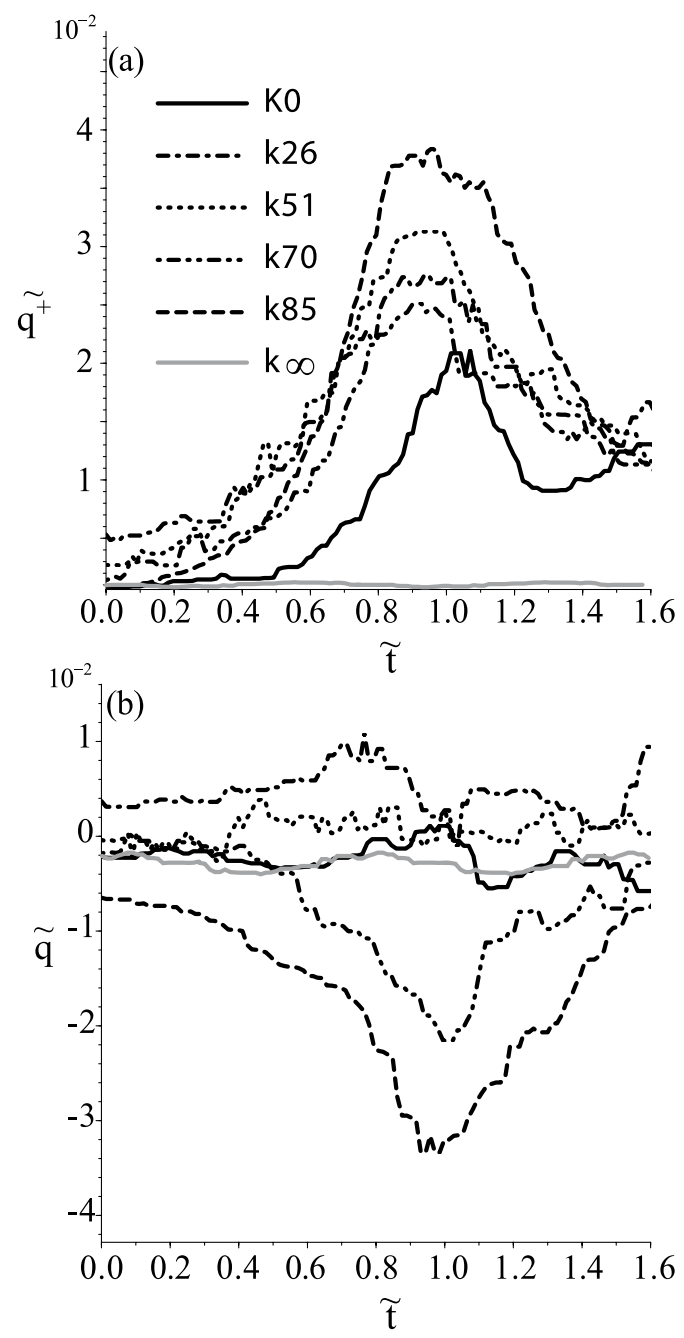

FIG. 12. Temporal evolution of the dimensionless flux exchange between the ambient fluid and the boundary. (a) Positive flux from Eq. (5); (b) total flux exchange.

Assuming the third error type is the dominant effect, positive flux is corrected by modifying positive velocities coming out of the foam at a height $z=1 \mathrm{~mm}$.

\section{DISCUSSION}

The experiments on the impact with a solid wall, reported in Sec. III, confirm the observation by previous researchers that the interaction goes through three phases, namely, (i) stretching due to the presence of its mirror image in the boundary, (ii) the generation of secondary vorticity of the opposite sign that forms a secondary ring that (iii) drives a rebound of the primary ring from the wall and causes the trajectory of the cores to loop. When permeable boundaries are used, all three of these phenomena are reduced as permeability increases. In particular, in the most permeable foam, $\mathrm{k} 85$, the ring does not rebound but continues to propagate forward and dissipates. This is confirmed by looking at the flux of primary vorticity across $z=1 \mathrm{~mm}$ computed as

$$
q_{\omega}=\Delta r \sum_{R_{i}} \omega\left(z, r_{i}, t\right) v_{i}\left(z, r_{i}, t\right) r_{i} \quad \omega>0.03 \omega_{\max },
$$

and made dimensionless by $\tilde{q}_{\omega}=q_{\omega} /\left(D_{t} V_{r}^{2}\right)$. The results obtained for the $\mathrm{k} 85$ case are shown in Figure 13 , where a clear change is visible after $\tilde{t}>\tilde{t}_{1}$, which is right after the frame at which the 


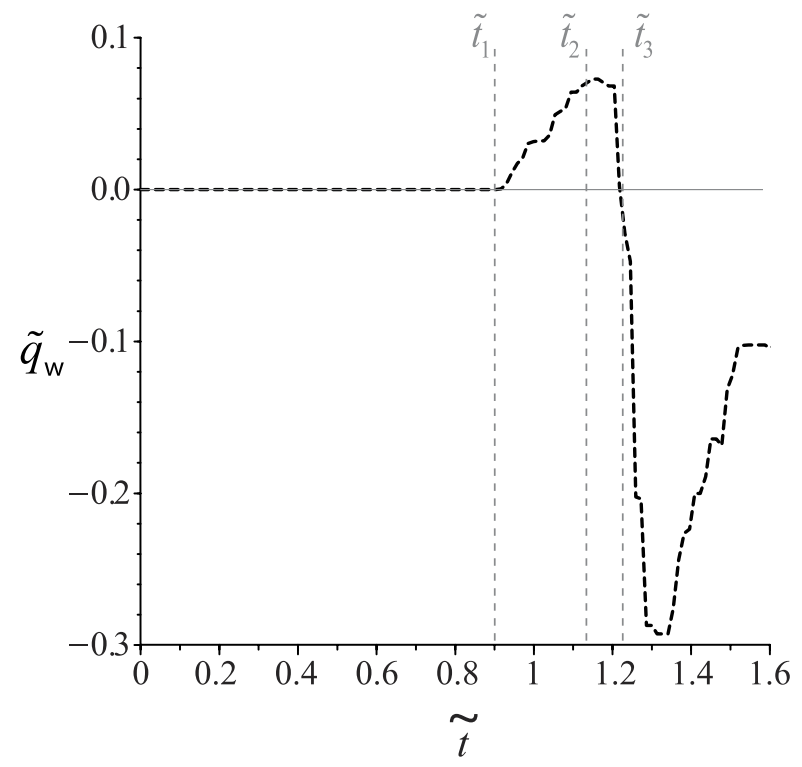

FIG. 13. Flux of primary vorticity through the $\mathrm{k} 85$ porous media, measured at $z=1 \mathrm{~mm}$.

primary vorticity starts decreasing in Figure 9(a). This also explains why the primary vorticity in the k85 permeable boundary decreases faster than the other boundary types: because the primary ring seems to enter inside the foam. This does not occur with the other cases as seen in Figure 8. Comparing the results with the $\mathrm{k} 0$ case, and bearing in mind that secondary vorticity in the $\mathrm{k} 85$ case is weaker, most of the decrease in vorticity shown in Figure 9 is due to the vortex ring penetrating/dissipating the foam. However, it is not clear which fraction of the circulation disappears through the porous boundary and this question is left for future investigations.

The permeable boundary results shown in this study are contrasted with two similar situations: bed sediments and thin porous grids. When comparing two different sediment bed layers (with different particle diameter and permeability), Bethke and Dalziel ${ }^{19}$ found that the trajectory followed by the vortex ring core was not noticeably affected by the bed permeability, although they report a weak exchange with the bed and an enhancement in the velocity immediately above the bed. The first of these observations contrasts with what we see here in Figure 6. The principal reason behind this difference is that the permeability of their porous media $\left(\kappa<9.4 \times 10^{-10} \mathrm{~m}^{2}\right)$ was between two and four orders of magnitude smaller than for the foams presented here, and consequently the flow into and out of the porous media was very much smaller and so had no measureable impact on the propagation of the ring. Also, our present results suggest the difference in approach distance would not have been measurable for such low permeabilities. To a good approximation, their porous boundaries were indistinguishable from solid boundaries except for the dynamics of the boundary layer that formed on it. However, with the substantially larger permeabilities used here, we see that the permeability has a clear influence on the vortex ring diameter expansion, the rebound, and the minimum height reached close to the boundary.

The evolution of the ring approaching boundaries with relatively high permeability is very similar to that of a vortex ring impinging on a thin porous grid (e.g., Refs. 20-22). Experimental setups differ with the research presented herein essentially at the position of the boundary and its thickness: they used very thin grids located far from the solid boundary, and the grid did not cover the entire plan form of the tank. Moreover, as with the porous grids, the pressure drop across the grid could be altered by changing either the porosity or the size of the wires. Therefore, the flux beyond the limit of the porous grids is substantially different from the flux inside thicker porous boundaries as the ones used in our experiments. However, it is worth to compare experiments because, to a good approximation, all our foams have the same porosity.

In the wire grid experiments, the decrease in vortex stretching and the changes in secondary vorticity formation are explained to be due to the loss of circulation of the primary ring, a feature also 
seen in Figure 9. According to Adhikari and Lim, ${ }^{20}$ this is caused by the self-induced flow around the axis of symmetry that forms a jet-like flow beyond the grid. In Naaktgeboren et al. ${ }^{21}$ the decrease in the impulse with more porous grids, as a reflection of the drag force exerted on the flow by the grids, was said to cause the reduction of the secondary vorticity and subsequent rebound. However, neither of these investigations take into account either the penetration of the boundary layer inside the porous media or the flux exchange between the downstream and upstream sides of the grid.

Recalling that one of the main differences between our experiments and the thin grid research already published is that the latter does not cover the whole plan form of the tank, the flux exchange between both sides of the grid is clearly influenced by this. From Figure 10, peak vertical velocities detected at the boundary increases with permeability. Therefore, the velocity coming out of the foam is higher in the coarsest case, $\mathrm{k} 85$, constraining the diameter of the ring and preventing the stretching. Combining both results, the reduced stretching when permeability increases is due to the smaller decrease in the flow near the axis of symmetry and the subsequent increase in flux exiting of the foam.

The macroscopic no-slip condition, satisfied in the solid boundary scenario and linked to the formation of the secondary vorticity, is found to disappear as permeability increases. This is consistent with the results from ${ }^{21}$ that relate the suppression of the secondary vorticity to the decrease in hydraulic impulse with grids of higher permeability. Bethke and Dalziel ${ }^{19}$ suggested the explanation for the apparent slip condition at the surface of a $1000 \mu \mathrm{m}$ bed sediment layer was, partly, the permeability of the layer itself. Although there is no pattern visible from the evolution of the secondary vorticity with permeability in Figure 9(b), the decrease in primary circulation as permeability increases shows how the no-slip boundary condition will also be affected by permeability.

Another phenomenon associated with different permeable beds is related to the boundary layer formed at the interface. As has already been noticed in Sec. III and according to Ref. 19, the maximum velocity just above the bed for a ring impinging a sediment bed layer evolves in the same way as for an inviscid vortex ring. Nevertheless, slow moving fluid that can be equated with a boundary layer is still present, as witnessed by what looks like the boundary layer separation that occurs even when the k85 foam is used. However, the detachment of this layer with the consequent formation of the secondary vortex ring differs from one boundary type to the other. As suggested by Figure 11, the maximum of the peak velocity just above the bed is reached earlier for higher $k$ values and coincides with the detachment of the boundary layer and the formation of the secondary vortex ring. The coherence of this secondary vortex, formed right after the ring reaches its minimum height, is lost due to two factors: the flux exchange and the extension of the boundary layer into the porous material found by Beavers and Joseph. ${ }^{25}$ When a more permeable boundary is used, the boundary layer is thicker and so higher stresses are needed to permit the entire detachment. This, added to the fact that the k85 boundary has a lower maximum bed velocity peak compared to less permeable boundary types, explains the poor coherence of the secondary vortex ring formed while approaching a high permeable boundary, as shown in Figure 8.

\section{CONCLUSIONS}

The experiments reported here explored the interaction of vortex rings with different permeable boundaries. Vortex rings impinging a solid boundary were also studied in order to compare the main characteristics of their motion towards the boundary with the permeable cases.

The foams used had a finite thickness of $25 \mathrm{~mm}$, except the coarsest $\mathrm{k} 85$ foam that was $50 \mathrm{~mm}$ thick. However, over this range, no influence of $h$ was found in the experiments, suggesting that the results presented herein can be extended to thicker permeable boundaries.

Results obtained using PIV showed how permeability affects the characteristics already found for vortex rings moving towards a solid boundary. Permeable boundaries changed the following.

(a) The diameter stretching: as permeability increases, the diameter of the primary ring is stretched less.

(b) The primary ring deceleration: the influence of the boundary decreases as permeability increases. 
(c) The secondary vortex ring formation: higher permeable boundaries presented a less coherent secondary ring with shorter life. This affects the negative stretching and the rebound of the primary ring. Moreover, the secondary vortex ring was formed earlier for higher permeable boundaries, mainly because primary vortex ring reached the interface faster.

The analysis of velocities close to the boundary in an attempt to quantify the fluxes between the free fluid and the porous layer, and the velocities within any boundary layer, revealed a significant influence of the height above the interface at which the measurements were taken. Unfortunately, we were unable to complete these measurements closer than $1 \mathrm{~mm}$ above the boundary due to the characteristics of the foam. While this was sufficiently close to analyse the vertical velocities, the analysis of the no-slip/slip boundary condition at the interface was more complicated. Maximum radial velocity results were less strongly affected by the no-slip boundary condition on the solid boundary than the experiment performed by ${ }^{19} \mathrm{Z}$ at which it is measured. Moreover, when secondary vorticity was computed, other errors regarding the choice of the interface level $(\tilde{Z}=0)$ proved to be important as well.

The radial velocity analysis showed an apparent evolution of the peak horizontal velocity from that associated with a no-slip boundary condition for a solid boundary to that of a slip boundary condition for permeable boundaries, despite the limitations imposed by the measurement height. This was confirmed with the previous analysis of the primary circulation evolution. This is broadly consistent with the suggestion by Ref. 19 for a particle layer.

Four of the five cases studied showed similar phases in the evolution of a vortex ring whether the boundary was solid or permeable. The exception to this was for the coarsest foam, $\mathrm{k} 85$, where the ring penetrated the foam. However, the vertical structure of the ring did not survive within the porous layer.

Finally, the research presented herein has shown that further investigation is needed for the interactions of vortex rings with permeable boundaries. For instance, azimuthal variations of the vortex ring characteristics were omitted from the analysis presented so far. Moreover, additional experiments are needed using different Reynolds numbers to see its influence on permeable boundaries and to determine the key dimensionless grouping that characterises the interaction.

\section{ACKNOWLEDGMENTS}

The experimental work presented herein was conducted during a four-months-long visit of A.M.C. to the Department of Applied Mathematics and Theoretical Physics at Cambridge University, UK. The financial support of the Ministerio de Educacion y Ciencia de España through Grant No. CGL 2009-13039 is gratefully acknowledged. The support of the UPC-Barcelona Tech University is also acknowledged.

${ }^{1}$ M. Staymates and G. Settles, "Vortex ring impingement and particle suspension,” Bull. Am. Phys. Soc. Vol. 1 (2005).

2 W. Stewart, "Helicopter behaviour in the vortex-ring conditions," Aeronautical Research Committee, Report and Memoranda No. 3117, 1951.

${ }^{3}$ S. Newman, R. Brown, J. Perry, S. Lewis, M. Orchard, and A. Modha, "Comparative numerical and experimental investigations of the vortex ring phenomenon in rotorcraft," in Proceedings of the 57th Annual Forum of the American Helicopter Society International, Washington, DC, USA, 09-11 May 2001.

${ }^{4}$ L. Kelvin, "The translatory velocity of a circular vortex ring," Philos. Mag. 33, 511-512 (1867).

${ }^{5}$ M. J. M. Hill, “On a spherical vortex,” Proc. R. Soc. London 55(331-335), 219-224 (1894).

${ }^{6}$ G. K. Batchelor, An Introduction to Fluid Dynamics (Cambridge University Press, UK, 1967).

7 J. Norbury, "A family of steady vortex rings," J. Fluid Mech. 57, 417-431 (1973).

${ }^{8}$ T. Maxworthy, "Some experimental studies of vortex rings," J. Fluid Mech. 81, 465-495 (1977).

${ }^{9}$ P. G. Saffman, "The approach of a vortex pair to a plane surface in inviscid fluid," J. Fluid Mech. 92, 497-503 (1979).

10 A. W. Cerra, Jr. and C. R. Smith, "Experimental observations of vortex ring interaction with the fluid adjacent to a surface," Interim Report Lehigh University, Bethlehem, PA, Department of Mechanical Engineering and Mechanics, (1983), Vol. 1.

${ }^{11}$ J. D. A. Walker, C. R. Smith, A. W. Cerra, and T. L. Doligalski, "The impact of a vortex ring on a wall," J. Fluid Mech. 181, 99-140 (1987).

${ }^{12}$ P. Orlandi and R. Verzicco, "Vortex rings impinging on walls: Axisymmetric and three-dimensional simulations," J. Fluid Mech. 256, 615 (1993).

${ }^{13}$ J. D. Swearingen, J. D. Crouch, and R. A. Handler, "Dynamics and stability of a vortex ring impacting a solid boundary," J. Fluid Mech. 297, 1-28 (1995).

${ }^{14}$ T. T. Lim, T. B. Nickels, and M. S. Chong, "A note on the cause of rebound in the head-on collision of a vortex ring with a wall," Exp. Fluids 12(1), 41-48 (1991). 
${ }^{15}$ R. J. Munro and S. B. Dalziel, “Attenuation technique for measuring sediment displacement levels,” Exp. Fluids 39, 602-613 (2005).

${ }^{16}$ N. Bethke, "Vortex ring interaction with a particle layer: Implications for sediment transport," Ph.D. thesis, (2008).

${ }^{17}$ R. J. Munro, N. Bethke, and S. B. Dalziel, "Sediment resuspension and erosion by vortex rings," Phys. Fluids 21, 046601 (2009).

${ }^{18}$ N. Masuda, J. Yoshida, B. Ito, T. Furuya, and O. Sano, "Collision of a vortex ring on granular material. Part I. Interaction of the vortex ring with the granular layer," Fluid Dyn. Res. 44(1), 015501 (2012).

${ }^{19}$ N. Bethke and S. B. Dalziel, "Resuspension onset and crater erosion by a vortex ring interacting with a particle layer," Phys. Fluids 24(6), 063301 (2012).

${ }^{20}$ D. Adhikari and T. T. Lim, “The impact of a vortex ring on a porous screen," Fluid Dyn. Res. 41(5), 051404 (2009).

${ }^{21}$ C. Naaktgeboren, P. S. Krueger, and J. L. Lage, "Interaction of a laminar vortex ring with a thin permeable screen," J. Fluid Mech. 707, 260-286 (2012).

22 J. T. Hrynuk, J. Van luipen, and D. Bohl, "Flow visualization of a vortex ring interaction with porous surfaces," Phys. Fluids 24, 037103 (2012).

${ }^{23}$ S. B. Dalziel, DigiFlow User Manual (2006).

${ }^{24}$ S. E. Widnall, D. B. Bliss, and C. Y. Tsai, "The instability of short waves on a vortex ring," J. Fluid Mech. 66(1), 35-47 (1974).

${ }^{25}$ G. S. Beavers and D. D. Joseph, "Boundary conditions at a naturally permeable wall," J. Fluid Mech. 30(1), 197-207 (1967).

${ }^{26}$ G. I. Taylor, "A model for the boundary condition of a porous material. Part 1," J. Fluid Mech. 49(02), 319 (1971).

${ }^{27}$ S. Richardson, "A model for the boundary condition of a porous material. Part 2," J. Fluid Mech. 49(02), 327-336 (1971). 